\title{
Static Parameter Detection and Identification of Power Grid Branch Based on PMU Measurement
}

\author{
Cui Yunfeng \\ North China Electric Power University, Beijing 102206, China \\ cyf09281992@qq.com
}

Keywords: static parameter, detection and identification, partition, local identification

\begin{abstract}
With the wide coverage of the WAMS in power system, it is possible to detect the static parameters of power grid branch with PMU measurement. The paper presents a method, that is ,by the reformation of state space and linear static state estimation. To improve branch parameter computation redundancy, KCL equations are added to the measurement equation which is presented in parameter space. Through a combination of global detection and local identification, combined with much statistical analysis of measurement profiles, the adverse effects brought by the lack of precision of PMU measurement can be minimized. Finally, a New England 39 buses test system and a practical example are presented to verify the effectiveness of the proposed algorithm.
\end{abstract}

\section{Introduction}

The accuracy of power grid branch parameters directly determines the effects of EMS analysis and application software, and also greatly affects the accuracy and reliability of power dispatching control system. The main reasons leading to the inaccuracy of network parameters is that power systems are growing and expanding too fast, which makes it hard for the power grid companies to master the parameters. The following analysis and decision-making software will be harmed ${ }^{[1-2]}$.The traditional static parameter identification method is to estimate the parameters by adding suspicious parameters to state variables ${ }^{[3-5]}$. But it's difficult to accurately locate the erroneous parameters before parameter estimation. Recently, with the increasing emphasis on the accuracy of the basic data, some new parameter identification methods are emerging. In reference [6-7], a method is proposed on condition that the voltage phase and current phase at both ends are measurable by PMU. Because of low redundancy, identification effect is not satisfactory. In literature [8-9], PMU measurement and SCADA measurement are both introduced to parameter estimation. Although the redundancy of the measurement is relatively high, simultaneous measurement sampling is difficult to meet, making it hard to implement in practical application.

In view of the existing problem of using PMU measurement to detect the error of branch parameter in [6], namely the low redundancy of calculation, node injection current equation in Cartesian coordinates is introduced to measurement equation to increase the redundancy. By replacing the state space, all the branch parameters are treated as state variables. The measurement equation is established based on branch current measurement and node injection current measurement. Information matrix can be built with PMU's voltage measurement, by which linear static state estimation can be carried on. Finally, the erroneous parameters are identified by the combination of global detection and local identification.

\section{State space conversion model}

Traditional, state estimation chooses the phase and angle (or real part and imaginary part) of bus voltage as state variables. While that's not suitable for branch parameter detection and identification. Therefore, the method of replacing the state space is adopted, and voltage is replaced by branch parameter as state variables.

The line model is presented with $\pi$ equivalent circuit. Line parameters include branch conductance g, susceptance b and susceptance to ground b0. As shown in Figure 1. 


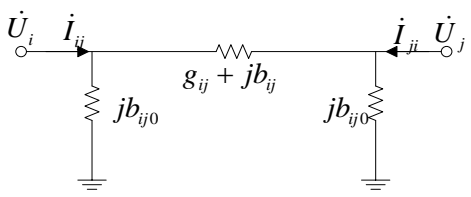

Figure $1 \pi$ model of transmission line

Selecting $g_{i j}, b_{i j}, b_{i j 0}$ as the state variables, the line's head and terminal branch current equations in Cartesian coordinates are shown in equations (1).

$$
\left[\begin{array}{c}
\operatorname{Re}\left(\dot{(}_{i j}\right) \\
\operatorname{Im}\left(\dot{I}_{i j}\right) \\
\operatorname{Re}\left(\dot{\mathrm{I}}_{j i}\right) \\
\operatorname{Im}\left(\dot{\mathrm{I}}_{j i}\right)
\end{array}\right]=\left[\begin{array}{ccc}
\left(e_{i}-e_{j}\right) & -\left(f_{i}-f_{j}\right) & -f_{i} \\
\left(f_{i}-f_{j}\right) & \left(e_{i}-e_{j}\right) & e_{i} \\
-\left(e_{i}-e_{j}\right) & \left(f_{i}-f_{j}\right) & -f_{j} \\
-\left(f_{i}-f_{j}\right) & -\left(e_{i}-e_{j}\right) & e_{j}
\end{array}\right]\left[\begin{array}{c}
g_{i j} \\
b_{i j} \\
b_{i j 0}
\end{array}\right]
$$

Where $e_{i}, f_{i}$ and $e_{j}, f_{j}$ are the real and imaginary parts of voltage phase respectively. Equations (1) can be written in block matrix form, namely:

$$
\left[\begin{array}{c}
I_{i j} \\
I_{j i}
\end{array}\right]=\left[\begin{array}{l}
H_{u . i j} \\
H_{u . j i}
\end{array}\right]\left[X_{L . i j}\right]
$$

Transformer's $\pi$ equivalent circuit is shown in Figure 2. Transformer parameters include branch conductance g, susceptance $b$ and two asymmetric susceptance to ground $b_{i 0} 、 b_{j 0}$.

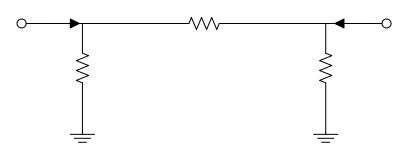

Figure $2 \pi$ model of transformer

Selecting $g 、 b 、 b_{i 0} 、 b_{j 0}$ as the state variables, the transformer's head and terminal branch current equations in Cartesian coordinates are shown in equations (2).

$$
\left[\begin{array}{c}
\operatorname{Re}\left(\dot{\mathrm{I}}_{i j}\right) \\
\operatorname{Im}\left(\dot{\mathrm{I}}_{i j}\right) \\
\operatorname{Re}\left(\dot{\mathrm{I}}_{j j}\right) \\
\operatorname{Im}\left(\dot{\mathrm{I}}_{j i}\right)
\end{array}\right]=\left[\begin{array}{cccc}
\left(e_{i}-e_{j}\right) & -\left(f_{i}-f_{j}\right) & -f_{i} & 0 \\
\left(f_{i}-f_{j}\right) & \left(e_{i}-e_{j}\right) & e_{i} & 0 \\
-\left(e_{i}-e_{j}\right) & \left(f_{i}-f_{j}\right) & 0 & -f_{j} \\
-\left(f_{i}-f_{j}\right)-\left(e_{i}-e_{j}\right) & 0 & e_{j}
\end{array}\right]\left[\begin{array}{c}
g \\
b \\
b_{i 0} \\
b_{j 0}
\end{array}\right]
$$

Equations (2) can be written in block matrix form, namely:

$$
\left[\begin{array}{l}
I_{i j} \\
I_{j i}
\end{array}\right]=\left[\begin{array}{c}
H_{u . i j} \\
H_{u . j i}
\end{array}\right]\left[X_{T . i j}\right]
$$

According to KCL, the injection current equation of each node can be listed. Together with the branch current equation, the PMU current measurement equation is composed, whose state variable is branch parameter, and whose coefficient matrix is PMU voltage measurement. The measurement equation can be briefly written: $\quad I=H_{u} x_{z}+v$

The branch parameters can be calculated by the weighted least square method, and the objective function is:

$$
\min J\left(\hat{x}_{z}\right)=\left(I-H_{u} \hat{x}_{z}\right)^{T} R^{-1}\left(I-H_{u} \hat{x}_{z}\right)
$$

The equation for the weighted least square linear state estimation is:

$$
\hat{x}_{z}=\left(H_{u}{ }^{T} R^{-1} H_{u}\right)^{-1} H_{u}{ }^{T} R^{-1} I
$$

Where gain matrix $G_{u}=H_{u}{ }^{T} R^{-1} H_{u}$

Matrix inversion can be avoided by solving the following equations:

$$
G_{u} \hat{x}_{\mathrm{z}}=H_{u}{ }^{T} R^{-1} I
$$

Now that the Jacobi matrix $H_{u}$, the weight matrix $R$ and the gain matrix $G_{u}$ are all constant matrix, the equation can be solved without iteration. By comparing the relative difference between the point estimation of the calculated values and the reference parameters of the system, the suspicious branch parameters can be detected. Measurement errors can be accumulated when calculate with all the network measurements in global detection. Therefore, after the detection of 
suspicious parameters, local estimation is applied to accurately identify the erroneous parameters.

\section{Method for detecting and identifying branch parameters of power grid}

The power grid parameter detection and identification algorithm mainly includes the following parts:

Global detection. First, several groups of PMU measurements are collected in a certain time window. KCL equations are added to the measurement equation to improve branch parameter computation redundancy, and linear static state estimation with reformed state space can be carried on. Followed by point estimation of the calculated value. When parameter with error exists, absolute difference and relative difference between the error parameter and its reference value will increase obviously, leaving us the criteria to find suspicious parameters.

Local partition. The advantages are as follows: other bad data can be isolated by partition, and its influence can be avoided; although the number of measurements used in parameter calculation are reduced, the redundancy of the parameter calculation in the local area is not reduced; for the relatively small calculation area and linear equation, computing efficiency will be improved. And depth first or breadth first method is used to search the connected branches, followed by computational area formation. That is topological partition method.

Local identification. According to the method mentioned above, computational areas are formed. Local identification can be applied with method which is similar to global detection. With the help of statistical analysis of sets of measurement profiles, errors in parameters can be identified.

\section{Case analysis}

IEEE model. New England 39 buses test system is introduced to verify the effectiveness of the proposed algorithm. The algorithm is realized by $\mathrm{C}++$ programming, and Gauss white noise is added to test the algorithm.

In the testing model, all the measurements are with $0.2 \%$ Gaussian noise. Method in reference [6] and method proposed in this paper are carried on respectively in the same testing model. Taking the resistance parameter $\mathrm{R}$ of line 16 and the reactance parameter $\mathrm{X}$ of the branch 12 of transformer as examples, each with 1000 measurement profiles. The results are shown in Figure 4 and 5.

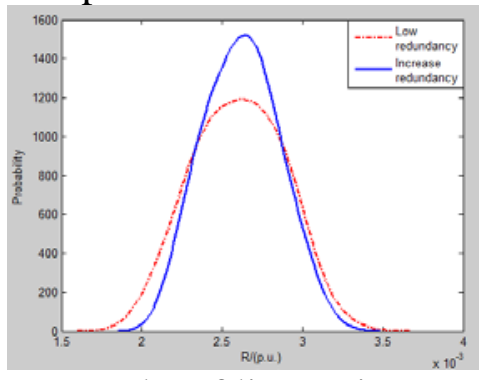

Figure 3 Comparison results of line resistance with two methods

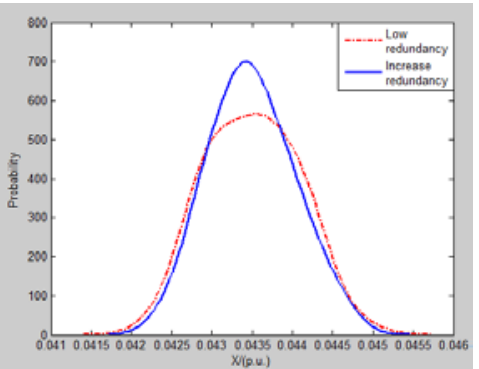

Figure 4 Comparison results of transformer reactance with two methods

From the analysis above, conclusions can be drawn: compared with the method in reference [6], parameters calculated by the method proposed in this paper are more close to the true value and with less variance. Thus, parameter detection and identification can be improved.

Figure 6 and 7 are the calculation results (1000 measurement profiles) with 2 partition method respectively, compared with global detection. The reactance $\mathrm{X}$ of branch 20 is taken as example. 


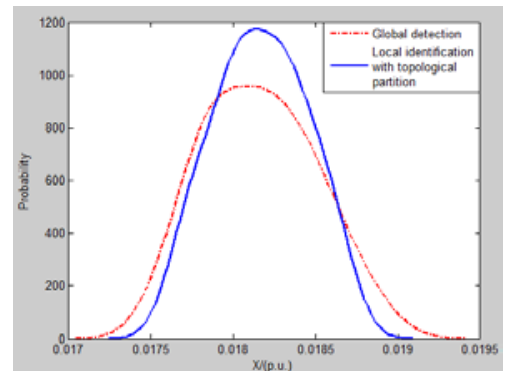

Figure 5 Results of local identification with topological partition

As we can see, the results of local identification are more close to the true value and with less variance. So this method can also improve the accuracy of the calculation, and thus parameter detection and identification can be improved.

In order to minimize the influence of accidental factors on parameter identification, it's advisable to use a number of measurement profiles to calculate. The stability of parameter calculation results with different number of measurement profiles are studied. In the test system, taking branch 45 as example, parameter calculation with different number of measurement profiles is performed with Gaussian white noise, followed by statistical analysis. Results are shown in Figure 8.

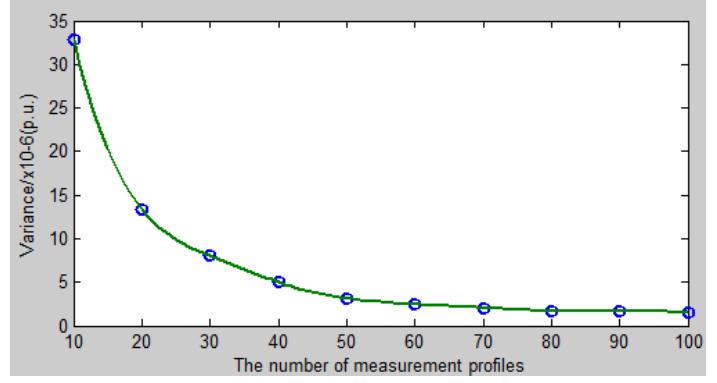

Figure 6 The variance of calculating results with different number of measurement profiles

As is shown in Figure 8, 80 measurement profiles are enough to obtain stable parameter calculation results. Note that, through the analysis and comparison of other branch parameters, although system structure had some influence on the results, the conclusion is basically the same.

Practical example. In this paper, the method is tested on part of the $500 \mathrm{kV}$ and $220 \mathrm{kV}$ bus networks of the North China Power Grid, and its system diagram is shown in Figure 9.

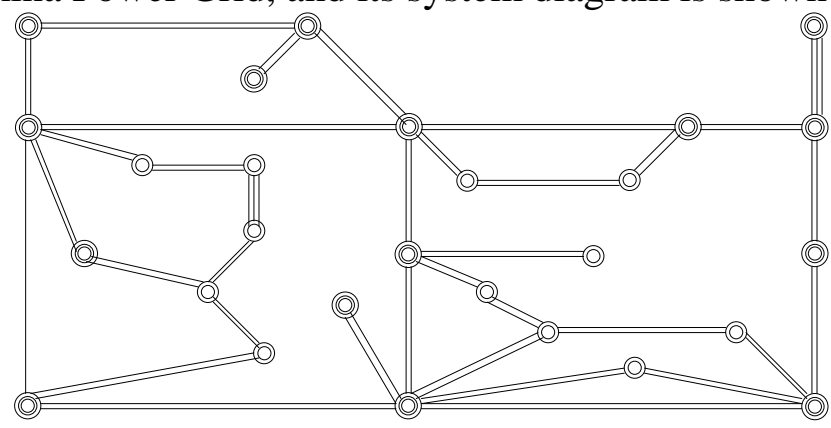

Figure 7 Part of system diagram of North China Power Grid

All the measurements are derived from BPA simulation. Gaussian white noise with a variance of $0.2 \%$ is added to measurements to simulate measurement error. Artificially set the error parameter and change the system reference value (p.u.). The resistance $\mathrm{R}$ of branch 4 (Angezhuang - Laoting) is changed from 0.00280 to 0.00250 ; the reactance $\mathrm{X}$ of branch 13 (Angezhuang - Luanxian) is changed from 0.00964 to 0.001200; the susceptance to ground B of branch 25 (Angezhuang Fengnan) is changed from -0.28570 to -0.30000 . The calculating results are shown in Figure 10 , Figure 11, Figure 12. On longitudinal axis, the positive value refers to transformer ratio, and the negative value refers to susceptance to ground. 


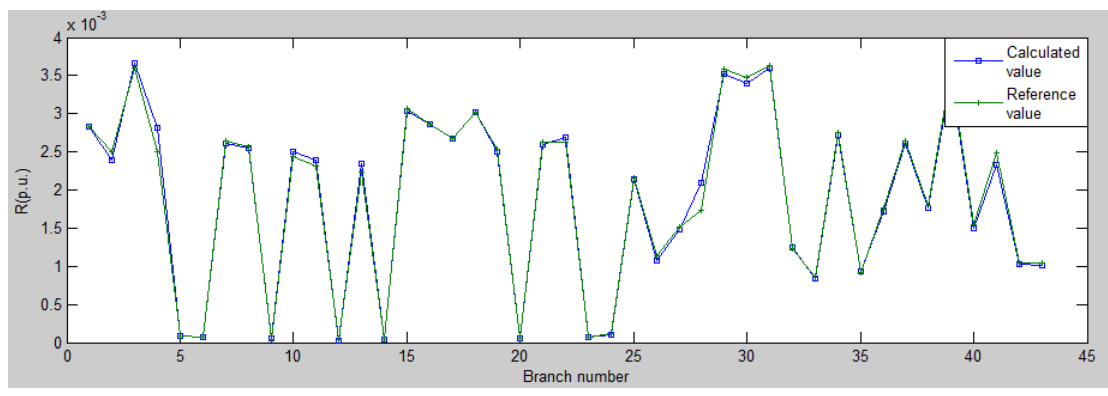

Figure 8 Comparison between the calculated value of branch resistance and reference value

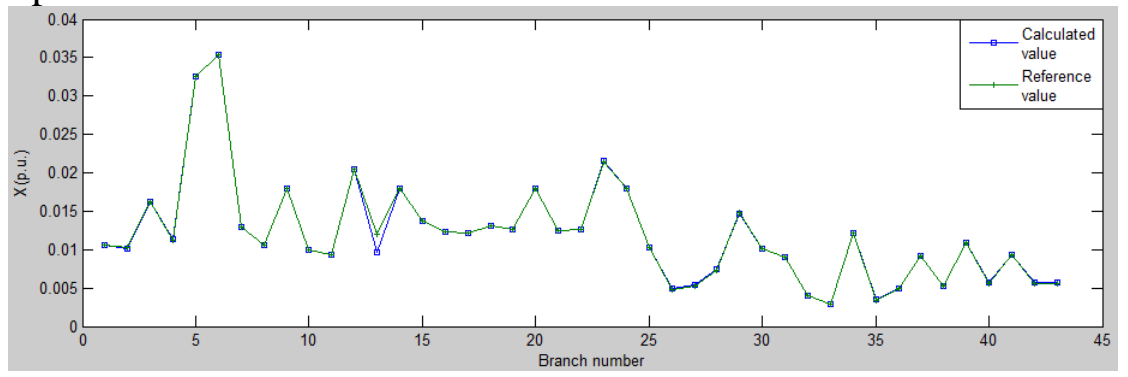

Figure 9 Comparison between the calculated value of branch reactance and reference value

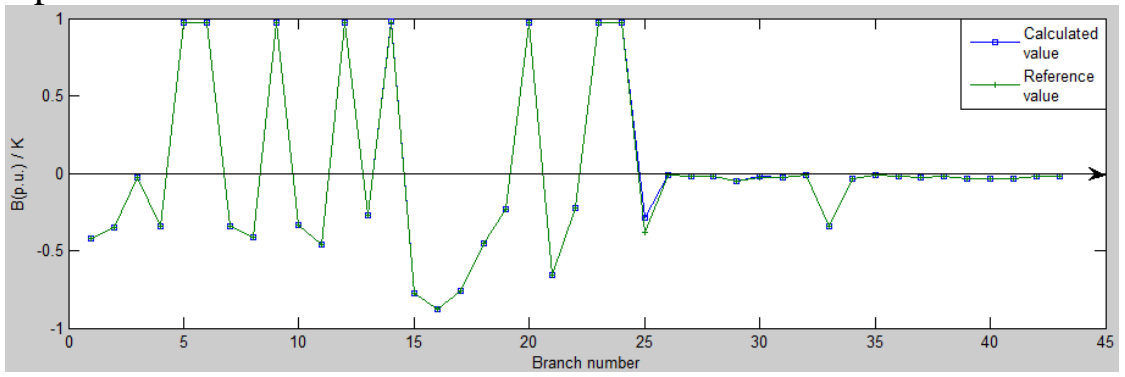

Figure 10 Comparison between the calculated value of susceptance to ground or transformer ratio and reference value

In this case, the criteria to identify suspicious parameters is set as: relative error $> \pm 5 \%$. And the suspicious parameters can be found according to this, namely: branch 4, branch 13, branch 25, branch 28. Their computational area can be formed respectively, followed by local identification. The results are shown in Table 3.

Table 1 Local identification results of suspicious parameters (p.u.)

\begin{tabular}{ccccccc}
\hline $\begin{array}{c}\text { Branch } \\
\text { number }\end{array}$ & $\begin{array}{c}\text { Calculated } \\
\text { R }\end{array}$ & $\begin{array}{c}\text { Reference } \\
\text { value R }\end{array}$ & $\begin{array}{c}\text { Calculated } \\
\text { X }\end{array}$ & $\begin{array}{c}\text { Reference } \\
\text { value X }\end{array}$ & $\begin{array}{c}\text { Calculated } \\
\text { B }\end{array}$ & $\begin{array}{c}\text { Reference } \\
\text { value B }\end{array}$ \\
\hline 4 & 0.002812 & 0.002500 & 0.001136 & 0.001120 & -0.34030 & -0.34000 \\
13 & 0.002286 & 0.002230 & 0.009392 & 0.012000 & -0.26946 & -0.26990 \\
25 & 0.002129 & 0.002160 & 0.010264 & 0.010260 & -0.28232 & -0.30000 \\
28 & 0.001829 & 0.001740 & 0.007376 & 0.007360 & -0.01909 & -0.01880 \\
\hline
\end{tabular}

During local identification, the criteria to identify parameters with error is set as: relative error $>$ $\pm 5 \%$. Through local identification, conclusions can be drawn: branch 28 is wrongly detected during global detection; the resistance $\mathrm{R}$ of branch 4 , the reactance $\mathrm{X}$ of branch 13 and the susceptance to ground $\mathrm{B}$ of branch 25 are erroneous parameters.

\section{Summary}

In this paper, a method with PMU measurement to detect and identify branch parameters is proposed. KCL equations are added to the measurement equation. And theory as state space conversion, linear static state estimation and network partition are involved. Through a combination of global detection and local identification, erroneous parameters are finally identified. Through $\mathrm{C}++$ programming and testing, the validity and practicability is verified. But the method requires that both ends of branch can get PMU measurements, making it hard to apply to some of networks. 


\section{References}

[1] W. E. Liu, F. Wu, M Lun, Estimations of parameter errors from measurement residuals in state estimation, IEEE Trans on Power Systems, 1992, 7(1): 81-89.

[2] A.G. Phadke, J.S. Thorp, Synchronized phasor measurements and their applications, NY, USA: Springer Press, 2008: 22-53.

[3] O. Alsac, N. Vempati, B. Stott, Montieelli A, Generalized state estimation, IEEE Trans on Power Systems, 1998, 13(3): 1069-1075.

[4] A. Olarte, H. Diaz, Transmission Line's Parameter estimation using state estimation algorithms, Power and Energy Society General Meeting, 2008: 1-7.

[5] K.A. Clements, P.W. Davis, Detection and identification of topology errors in electric power systems, IEEE Transactions on Power Systems, 1988, 3(4): 1748-1753.

[6] X. Zhao, H. Zhou, D. Shi, On-line PMU-based transmission line parameter identification, CSEE Journal of Power and Energy Systems, 2015, 1(2): 68-74.

[7] R.E. Wilson, A. Gary, Calculation of transmission line parameters from synchronized measurements, Electric Machines and Power Systems, 1999, 27(12): 1269-1278.

[8] L. Ding, T. Bi, D. Zhang, Transmission line parameters identification based on moving-window TLS and PMU data, in Proc. IEEE Int. Conf. Adv. Power Syst. Automation Protection, Beijing, China, 2011(3), pp. 2187-2191.

[9] M.R. Castillo, J.B. London, Off-line detection, identification and correction of branch parameter errors using SCADA and synchronized phasor measurements, Transmission \& Distribution Conference and Exposition - Latin America, 2014 IEEE PES,2014, pp. 1-5. 